\title{
Coexisting conical bipolar and equatorial outflows from a high-mass protostar
}

\section{Citation}

Greenhill, L. J., C. R. Gwinn, C. Schwartz, J. M. Moran, and P. J. Diamond. 1998. Nature 396 (6712) (December 17): 650-653. doi:10.1038/25299.

\section{Published Version}

$10.1038 / 25299$

\section{Permanent link}

http://nrs.harvard.edu/urn-3:HUL.InstRepos:32714413

\section{Terms of Use}

This article was downloaded from Harvard University's DASH repository, and is made available under the terms and conditions applicable to Other Posted Material, as set forth at http:// nrs.harvard.edu/urn-3:HUL.InstRepos:dash.current.terms-of-use\#LAA

\section{Share Your Story}

The Harvard community has made this article openly available.

Please share how this access benefits you. Submit a story.

\section{Accessibility}




\section{Coexisting conical bipolar and equatorial outflows from}

\section{a high-mass protostar}

L. J. Greenhill*, C. R. Gwinn ${ }^{\dagger}$, C. Schwartz ${ }^{\ddagger}$, J. M. Moran*, P. J. Diamond]

The $\mathrm{BN} / \mathrm{KL}$ region in the Orion molecular cloud is an archetype in the study of the formation of stars much more massive than the Sun. This region contains luminous young stars and protostars, but it is difficult to study because of overlying dust and gas. Our basic expectations are shaped to some extent by the present theoretical picture of star formation, the cornerstone of which is that protostars acrete gas from rotating equatorial disks, and shed angular momentum by ejecting gas in bipolar outflows. The main source of the outflow in the $\mathrm{BN} / \mathrm{KL}$ region 胭自 may be an object known as radio source $\mathrm{I}^{\mathrm{H}}$, which is commonly believed to be surrounded by a rotating disk of molecular material 明䀦. Here we report high-resolution observations of silicon monoxide $(\mathrm{SiO})$ and water maser emission from the gas surrounding source I; we show that within 60 AU (about the size of the Solar System), the region is dominated by a conical bipolar outflow, rather than the expected disk. A slower outflow, close to the equatorial plane of the protostellar system, extends to radii of 1,000 AU.

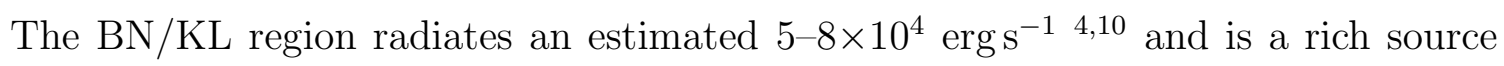
of molecular line emissionen embedded within the Orion Molecular Cloud (OMC-1). Two molecular outflows originate in BN/KL, relatively close to source $\mathbf{I}$, a low-velocity $\left(18 \mathrm{~km} \mathrm{~s}^{-1}\right)$ outflow to the northeast-southwest 11.12 , and an orthogonal high-velocity

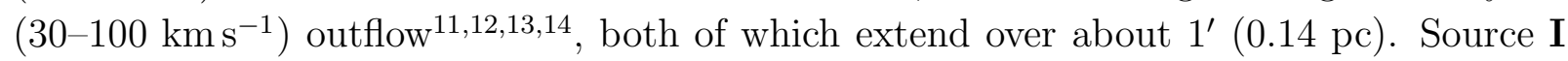
has a broad-band radio spectrum consistent with an H II region about $60 \mathrm{AU}$ in diameter国, though it has not yet been specifically detected at infrared wavelengths. Masers are often associated with massive young stars, and many are broadly distributed across BN/KL (masers are the microwave equivalent of lasers). However, a distinct population of $\mathrm{SiO}$ and $\mathrm{H}_{2} \mathrm{O}$ masers is clustered around source $\mathbf{1 2}$. To understand better the physical nature of source $\mathbf{I}$ and its relationship to the outflows in BN/KL, we have mapped these masers with high spectral and angular resolution. We resolve the angular structure of the $\mathrm{SiO}$ emitting

\footnotetext{
${ }^{*}$ Center for Astrophysics, 60 Garden Street, Cambridge, MA 02138.

${ }^{\dagger}$ University of California, Santa Barbara, CA 93106.

${ }^{\ddagger}$ Maria Mitchell Observatory, Nantucket, MA 02554. Currently Colby College, Waterville, ME 04901.

$\S$ National Radio Astronomy Observatory, PO Box O, Socorro, NM 87801.
} 
region in detail at each observed line-of-sight velocity, in contrast to previous observations with connected-element radio interferometers 0 .

Maser sources are valuable tracers in molecule-rich regions because they comprise many compact, high surface-brightness, spatially distinct points of radiation (spots), with various line-of-sight velocities. The spots are test "particles" tracing the three-dimensional velocity field of the material in which they are embedded. Maser amplification requires relatively small gradients in line-of-sight velocity to achieve long gain-path lengths. To pump the maser levels by collisions, the gas temperature must also be at least a few hundred degrees, and the density orders of magnitude greater than that sampled by thermal molecular emission, i.e., $\mathrm{n}_{H_{2}} \gtrsim 10^{8} \mathrm{~cm}^{-3}$. Maser emission is a selective tracer but in star forming regions, it can in principle occur in either outflows or disks17.

We observed emission from the $v=1, J=1-0$ transition of $\mathrm{SiO}(43.12208 \mathrm{GHz}$ rest frequency) toward the BN/KL region on 9 July 1995 with the Very Long Baseline Array (VLBA) of the NRAO. The data were processed with the VLBA correlator, which provided $0.22 \mathrm{~km} \mathrm{~s}^{-1}$ wide spectral channels, and reduced with standard techniques $1 \mathrm{~g}$. Within $30 \mathrm{~km} \mathrm{~s}^{-1}$ of the $5 \mathrm{~km} \mathrm{~s}^{-1}$ systemic velocity $\mathrm{l}^{\mathrm{b}}$ of the region near source $\mathbf{I}$, we detected 1,212 maser spots with flux densities between 0.09 and $300 \mathrm{Jy}$.

Spectroscopic images show that the $\mathrm{SiO}$ maser emission is confined to four regions that can be envisioned as marking the arms of an $\mathbf{X}$, subtending about 0.'25 (Figure 1a). We propose that the $\mathbf{X}$ marks the intersection of two outflow cones with the plane of the sky and separates the low-velocity (northeast-southwest) and high-velocity (northwest-southeast) outflows in BN/KL. Source I was not detected by us because its surface brightness is too low. However, to estimate the position of source $\mathbf{I}$ on our maps, we compared the flux-weighted emission centroid for each VLBA spectral channel to the earlier, lower resolution $(0,25)$ map of Menten \& Reid目 who measured the position of source $\mathbf{I}$ relative to the maser emission centroids with 0.'01 uncertainty, using the Very Large Array (VLA). The relative positions of the dominant clumps of masers in the two maps agree to $0 . \prime 01$. Source $\mathbf{I}$ lies at the center of the $\mathbf{X}$, within the measurement errors (Figure 1a). The innermost $\mathrm{SiO}$ maser features are separated by about the 60 AU diameter of the source I H II region.

We have added to our study archived $\mathrm{H}_{2} \mathrm{O}$ maser data collected on 25 August 1983 with the VLA (Figure 1b), as well as published maps $1 \mathrm{~g}$ of the $\mathrm{H}_{2} \mathrm{O}$ maser distribution on 5 August 1991. The distribution of the $\mathrm{H}_{2} \mathrm{O}$ masers is elongated in the direction of the low-velocity outflow in BN/KL. Over eight years, the length of the distribution grew by about 0 "2. We measured proper motions for 19 individual maser features that persisted over the eight year period (Figure 1b). The motions are primarily parallel to the axis of the low-velocity outflow, and the average transverse motion corresponds to about $20 \mathrm{~km} \mathrm{~s}^{-1}$, 
approximately the speed of the large scale low-velocity outflow in BN/KI 12.

The previous widely accepted model, in which the $\mathrm{SiO}$ masers lie in a rotating, expanding, and inclined disk 8 国, does not fit our high-resolution VLBA maps. One attraction of this model was that the disk could feasibly extend to larger radii, explaining the low-velocity $\mathrm{BN} / \mathrm{KL}$ outflow. In such a disk, the $\mathrm{SiO}$ emission is expected to trace dynamic surfaces where the derivative of the line-of-sight velocity is small, so that the maser gain is high. For most rotation laws, each arm of the $\mathbf{X}$ would be expected to show a velocity gradient along its length; but three of four do not. Moreover, the distribution of $\mathrm{SiO}$ masers that we observe shows approximate mirror symmetry in position and velocity about a northwest-southeast axis, which constrains the magnitude of any rotation to be less than a few $\mathrm{km} \mathrm{s}^{-1}$. If the disk is gravitationally bound to the protostar, then this rotation speed, at 25-60 AU radius, constrains the protostellar mass to be less than $1 \mathrm{M}_{\odot}$. If radial forcing enlarges the disk (e.g., a wind) from an initially bound state (with about 1 AU radius), then conservation of angular momentum requres a stellar mass less than a few $\mathrm{M}_{\odot}$. These relatively small stellar masses are inconsistent with the presence of an H II region.

We propose that the $\mathrm{SiO}$ maser emission outlines the limbs of a bipolar, conical, high-velocity flow 25-60 AU from the protostar (i.e., the $\mathbf{X}$ ), and that the $\mathrm{H}_{2} \mathrm{O}$ masers lie in a low-velocity flow in an equatorial band bisecting the high-velocity flow (Figure 1c). The position angles of the cones are $-40^{\circ}$ and $150^{\circ}$, with opening angles of $60^{\circ}$ and $80^{\circ}$, respectively. For an outflow axis close to the plane of the sky and a finite cone wall thickness, the longest maser gain paths naturally lie along the limbs of the cones. The 10-15 $\mathrm{km} \mathrm{s}^{-1}$ difference in mean Doppler velocity between the red- and blue-shifted $\mathrm{SiO}$ masers may be explained if the cones are tipped out of the plane of the sky. The known distribution of $\mathrm{H}_{2}$ emission in $\mathrm{BN} / \mathrm{KL} 20$ shows two lobes with line-of-sight velocities Doppler shifted by more than $30 \mathrm{~km} \mathrm{~s}^{-1}$ that subtend the openings of the cones. The northwest lobe is equally prominent in red- and blue-shifted emission, which suggests that the inclination is small. (Blue-shifted emission from the southeast lobe appears to be blocked by a dense foreground cloud $15,21$.

The dense gas in which the $\mathrm{SiO}$ masers lie is probably not gravitationally bound to the protostar because there is no detectable rotation. The $\mathrm{SiO}$ maser emission probably arises at a steady-state shock interface, perhaps between a faster and a slower component of the stellar outflow. Such shocks serve as efficient mechanisms that collisionally pump $\mathrm{SiO}$ masers and reduce depletion of gas-phase $\mathrm{SiO}$ onto dust grains. To quantify the lifetime of the visible structure, we compared the present $\mathrm{SiO}$ maser maps with a partially complete VLBI map obtained in 198522. The loci of red-shifted masers have remained fixed with respect to source $\mathbf{I}$ to about $0{ }^{\prime \prime} 01$ over 10.3 years, which corresponds to a proper motion of 
less than 4.5 AU or $2 \mathrm{~km} \mathrm{~s}^{-1}$. We speculate that collimation of the bipolar outflow begins at radii less than $25 \mathrm{AU}$, perhaps inside the $\mathrm{H}$ II region, because source $\mathbf{I}$ lies close to the apices of the outflow cones.

We fit the $\mathrm{H}_{2} \mathrm{O}$ maser positions and Doppler velocities to a constant-velocity outflow centered on source $\mathbf{I}$, where the $\mathrm{H}_{2} \mathrm{O}$ masers are constrained to lie on a toroidal surface, as suggested qualitatively by the curvature traced by several $\mathrm{H}_{2} \mathrm{O}$ features (Figure $1 \mathrm{c}$ ). This surface may be where large-diameter dust grains begin to form and to be accelerated by radiation pressure. (Dust condensation and $\mathrm{H}_{2} \mathrm{O}$ maser excitation together require temperatures of 400-1,000 K. If this material is in thermal equilibrium, then the luminosity of source I would be $\gg 10^{4} \mathrm{~L}_{\odot}$.) The torus has the following parameters: mean outer radius $360 \mathrm{AU}$, thickness 270-320 AU, outflow velocity $13 \mathrm{~km} \mathrm{~s}^{-1}$, and rotation less than a few $\mathrm{km} \mathrm{s}^{-1}$. The torus is tipped down about $10^{\circ}$ with respect to the line of sight, as evidenced by the southeasterly position bias of the distribution of blue-shifted $\mathrm{H}_{2} \mathrm{O}$ masers. (The masers lie close to the equator.) We assume that the axes of the high-velocity outflow and the torus are aligned (Figure 1c), from which we infer that the high-velocity outflow is at least $50 \mathrm{~km} \mathrm{~s}^{-1}$. This limit is consistent with the observed three-dimensional motions of Herbig-Haro objects in $\mathrm{BN} / \mathrm{KL}_{2}, \mathrm{~L} 4$ and the Doppler velocities of thermal $\mathrm{CO}$ and $\mathrm{SiO}$ emission 11 . We note that the $\mathrm{SiO}$ maser emission from the limbs of the cones suggests material flowing away from the observer toward the northwest, opposite that expected given inclination of the torus. However, we consider that the masers trace gas selectively. For small inclinations, optimal amplification can occur with the opposite sense of Doppler shift (i.e., red- vs blue-shift) from gas along the axes of the cones, depending on the radial and angular dependence of the outflow velocity.

We conclude that the equatorial region is dominated by outflowing material and not by (rotating) natal material. We may extend this outflow to radii of up to 1,000 AU by considering the previously mapped distribution of $\mathrm{v}=0 \mathrm{SiO}$ emission 24 , to which three components contribute: high-velocity thermal, low-velocity thermal, and low-velocity maser emission. Both low-velocity components overlie the $\mathrm{H}_{2} \mathrm{O}$ maser distribution in velocity and sky position within $350 \mathrm{AU}$ of source $\mathbf{I}$. The brightness distribution is asymmetric with respect to source $\mathbf{I}$, as is the $\mathrm{H}_{2} \mathrm{O}$ maser torus. On the smaller side of the torus, stronger $\mathrm{SiO}$ emission coincides with a richer distribution of $\mathrm{H}_{2} \mathrm{O}$ masers, which is consistent with a density gradient in the surrounding medium, as seen at 8.7 and $12.4 \mu \mathrm{m}$ 胭. Although the apparent mixing of $\mathrm{H}_{2} \mathrm{O}$ maser and $\mathrm{SiO}$ emission is difficult to understand (since silicate-grain formation depletes gas-phase $\mathrm{SiO}$ ), we note that similar mixing is apparent in the envelopes of late-type stars $25,26$.

We note three propitious alignments of structure that strengthen the case for the 
proposed model. First, no $\mathrm{H}_{2} \mathrm{O}$ masers lie within the high-velocity flow demarcated by the $\mathrm{SiO}$ masers (Figure 1c). This flow is probably too hot or turbulent to support maser action. Second, the four components of IRc22 shown in Figure 2 subtend the opening angle of the northern outflow cone. They may have a direct line of sight to the protostar and may be heated by it or by interaction with the high-velocity flow. Infrared light from the southeastern outflow is blocked by extinction in a foreground cloud core 25.21 . Third, the magnetic field direction inferred from dust emission at $100 \mu \mathrm{m}$ and $3 \mathrm{~mm}$ wavelength is parallel to the high-velocity outflow axis28.29. This suggests that the formation of the protostar and the alignment of the outflow axis may have been affected by the broad field distribution in OMC-1.

We argue that the masers clustered around source I provide evidence for a twocomponent outflow from a young star. In the context of star formation theory for high-mass stars, no current model readily explains wide-angle collimation of a high-velocity bipolar outflow within tens of $\mathrm{AU}$ of a protostar and a simultaneous low-velocity equatorial outflow. Magnetohydrodynamic (MHD) mechanisms such as X-winds 30 and disk winds 31 may be reasonable candidates. Alternatively, a spherical stellar wind that mixes with and ablates an (unseen) accretion disk up to about $350 \mathrm{AU}$ in radius might generate a slow, dense equatorial wind, but this would not explain the observed conical structures. In addition, the presence of an H II region may preclude a disk. If accretion and outflow processes are linked, then the birth of an H II region could dissipate the observed equatorial outflow in about 100-200 years. Based on the alignment of the outflows from source $\mathbf{I}$ with other structures in $\mathrm{BN} / \mathrm{KL}$, we suggest that source $\mathbf{I}$ drives a substantial portion of the bulk motion in BN/KL, though other sources, such as $\mathbf{n}$ (Figure 2), rightly draw attention. 6 . We anticipate that VLBA maps of $\mathrm{SiO}$ and $\mathrm{H}_{2} \mathrm{O}$ maser proper motions and $\mathrm{SiO}$ maser polarizations will clarify the structure, dynamics, and magnetic field geometry in this region, which will help resolve the debate about sources of energy and outflow in BN/KL.

\section{REFERENCES}

1. Kleinmann, D. E., \& Low, F. J. Discovery of an infrared nebula in Orion. Astrophys. J. 149, L1-L4 (1967).

2. Genzel, R. \& Stutzki, J. The Orion molecular cloud and star-forming region. Ann. Rev. Astron. Astrophys. 27, 41-85 (1989).

3. Gezari, D. Y. Mid-infrared imaging of Orion BN/KL: astrometry of IRc2 and the SiO maser. Astrophys. J. 396, L43-L47 (1992).

4. Gezari, D. Y. \& Backman, D. E. 4.8-20 micron imaging of Orion BN/KL: II. A new look at luminosity sources and the role of IRc2. Astroph. \& Space Sci. 224, 45-52 
(1995).

5. Menten, K. M. \& Reid, M. J. What is powering the Orion Kleinmann-Low infrared nebula? Astrophys. J. 445, L157-L160 (1995).

6. Churchwell, E., Wood, D. O. S., Felli, M., \& Massi, M. Solar system-sized condensations in the Orion Nebula. Astrophys J. 321, 516-519 (1987).

7. Plambeck, R. L., Wright, M. C. H. \& Carlstrom, J. E. Velocity structure of the Orion-IRc2 SiO maser: evidence for an $80 \mathrm{AU}$ diameter circumstellar disk. Astrophys. J. 348, L65-L68 (1990).

8. Wright, M. C. H., Plambeck, R. L., Mundy, L. G. \& Looney, L. W. SiO emission from a 1,000 AU disk in Orion KL. Astrophys. J. 455, L185-L188 (1995).

9. Barvainis, R. The polarization of the $\mathrm{SiO}$ masers in Orion: maser emission from a rotating, expanding disk? Astrophys. J. 279, 358-362 (1984).

10. Thronson, H. A. Jr., et al. The Orion star-forming region: far-infrared and radio molecular observations. Astron. J. 91, 1350-1356 (1986).

11. Wright, M. C. H., Plambeck, R. L. \& Wilner, D. J. A multiline aperture synthesis study of Orion-KL. Astrophys. J. 469, 216-237 (1996).

12. Genzel, R., Reid, M. J., Moran, J. M. \& Downes, D. Proper motions and distances of $\mathrm{H}_{2} \mathrm{O}$ maser sources. I. The outflow in Orion-KL. Astrophys. J. 244, 884-902 (1981).

13. Allen, D. A. \& Burton, M. G. Explosive ejection of matter associated with star formation in the Orion nebula. Nature 363, 54-56 (1993).

14. Jones, B. F. \& Walker, M. F. Proper motions of Herbig-Haro objects. VI. The M42 HH objects. Astron. J. 90, 1320-1323 (1985).

15. Plambeck, R. L., Wright, M. C. H., Mundy, L. G., \& Looney, L. W. Subarcsecondresolution $86 \mathrm{GHz}$ continuum maps of Orion KL. Astrophys J. 455, L189-L192 (1995).

16. Morita, K.-I., Hasegawa, T., Ukita, N., Okumura, S. K. \& Ishiguro, M. Accurate positions of $\mathrm{SiO}$ masers in active star forming regions: Orion-KL, W51-IRS2, and Sgr-B2 MD5. Pub. Astron. Soc. Japan 44, 373-380 (1992).

17. Torrelles, J. M., et al. Radio Continuum-H2O Maser Systems in NGC 2071: H2O Masers Tracing a Jet (IRS 1) and a Rotating Proto-planetary Disk of Radius 20 AU (IRS 3). Astrophys. J. 505, 756-765 (1998). 
18. Zensus, J. A., Diamond, P. J. \& Napier, P. J., eds. Very Long Baseline Interferometry and the VLBA (Astron. Soc. Pacific, San Francisco, 1995).

19. Gaume, R. A., Wilson, T. L., Vrba, F. J., Johnston, K. J. \& Schmid-Burgk, J. Water masers in Orion. Astrophys. J. 493, 940-949 (1998).

20. Sugai, H., Kawabata, H., Usuda, T., Inoue, M. Y., Kataza, H. \& Tanaka, M. Velocity field of the Orion-KL region in molecular Hydrogen emission. Astrophys. J. 442, 674-678 (1995).

21. Migenes, V., Johnston, K. J., Pauls, T. A. \& Wilson, T. L. The distribution and kinematics of ammonia in the Orion-KL nebula: high-sensitivity VLA maps of the $\mathrm{NH}_{3}(3,2)$ line. Astrophys. J. 347, 294-301 (1989).

22. Greenhill, L. J. et al. in The Impact of VLBI on Astrophysics and Geophysics (eds Reid, M. J. \& Moran, J. M.) 253-254 (Kluwer, Dordrecht, 1988).

23. $\mathrm{Hu}, \mathrm{X}$. Kinematic Studies of Herbig-Haro Objects in the Orion Nebula. Astron. J. 112 2712-2717 (1996).

24. Chandler, C. J. \& De Pree, C. G. Vibrational ground-state $\mathrm{SiO} J=1-0$ emission in Orion IRc2 imaged with the VLA. Astrophys. J. 455, L67-L71 (1995).

25. Lucas, R., et al. Interferometric observations of $\mathrm{SiO} v=0$ thermal emission from evolved stars. Astron. \& Astrophys. 262, 491-500 (1992).

26. Danchi, W. C., Bester, M., Degiacomi, C. G., Greenhill, L. J., \& Townes, C. H. Characteristics of dust shells around 13 late-type stars. Astron. J. 107, 1469-1513 (1994).

27. Dougados, C., Lena, P., Ridgway, S. T., Christou, J. C. \& Probst, R. G. Near-infrared imaging of the Becklin-Neugebauer-IRc2 region in Orion with subarcsecond resolution. Astrophys. J. 406, 112-121 (1993).

28. Schleuning, D. A. Far-infrared and submillimeter polarization of OMC-1: evidence for magnetically regulated star formation. Astrophys. J. 493, 811-825 (1998).

29. Rao, R., Crutcher, R. M., Plambeck, R. L. \& Wright, M. C. H. High-resolution millimeter-wave mapping of linearly polarized dust emission: magnetic field structure in Orion. Astrophys. J. 502, L75-L78 (1998). 
30. Shu, F. H., Najita, J., Ostriker, E. C. \& Shang, H. Magnetocentrifugally driven flows from young stars and disks. V. Asymptotic collimation into jets. Astrophys. J. 455, L155-L158 (1995).

31. Wardle, M., \& Königl, A. The structure of protostellar accretion disks and the origin of bipolar flows. Astrophys. J. 410, 218-238 (1993).

32. Garay, G., Moran, J. M. \& Haschick, A. D. The Orion-KL super water maser. Astrophys. J. 338, 244-261 (1989).

We thank Mark Reid for assistance in recovering his 1983 VLA data. We thank Catherine Dougados and Karl Menten for permission to use the infrared and radio images presented in Figure 2. CS was supported by the NSF REU program. C.R.G. acknowledges support of the National Science Foundation. The National Radio Astronomy Observatory is a facility of the U.S. National Science Foundation operated under cooperative agreement by Associated Universities, Inc. 


\section{CAPTIONS}

Figure 1a The $v=1, J=1-0 \mathrm{SiO}$ maser distribution in the $\mathrm{BN} / \mathrm{KL}$ region, obtained with the VLBA. Unlike previous maps made with connected-element radio interferometers, this one resolves the angular structure of the $\mathrm{SiO}$ emitting region at each observed line-of-sight velocity. The cross represents the centroid and position uncertainty for continuum source I with respect to the masers. The double arrows indicate the direction of the proposed high-velocity outflow from source $\mathbf{I}$, and the approximate direction of the high-velocity outflow in BN/KL. Symbol color indicates Doppler velocity in $\mathrm{km} \mathrm{s}^{-1}$ with respect to the Local Standard of Rest, as indicated by the colored bar. The dark bar indicates $25 \mathrm{AU}$, for an assumed distance of $450 \mathrm{pc}$. The interferometer beam is $0.40 \times 0.16$ milliarcseconds $(0.18 \times 0.07 \mathrm{AU})$ and the relative position uncertainties for the masers are smaller than the plotted symbols. We referenced the data to the strong emission at $-3.4 \mathrm{~km} \mathrm{~s}^{-1}$ (at the origin) to stabilize the VLBA against atmospheric fluctuations along the line of sight. Deconvolution of the instrumental response required special attention because most images contained complex structure, and because of the proximity of Orion to the celestial equator. From our VLBA data, we estimate that the origin of the map is $\alpha_{2000}=5^{h} 35^{m} 14^{s} .5101 \pm 0.0003, \delta_{2000}=-05^{\circ} 22^{\prime} 300^{\prime \prime} 63 \pm 0.05$.

1b The distribution of $\mathrm{H}_{2} \mathrm{O}$ masers in $\mathrm{BN} / \mathrm{KL}$ within $2^{\prime \prime}$ of source $\mathbf{I}$, observed with the VLA in 1983, at 0 "'1 resolution. The map of the SiO maser emission is shown within the dashed contour. The cross marks the position of source $\mathbf{I}$. The registration uncertainty between the $\mathrm{H}_{2} \mathrm{O}$ and $\mathrm{SiO}$ distributions is about 0. ".05, limited by uncertainty in the astrometric position of each with respect to the extragalactic radio reference frame. Arrows attached to the $\mathrm{H}_{2} \mathrm{O}$ maser spots show the estimated relative proper motions over 8 years. The mean motion has been subtracted to account for motion of the reference emission. The double arrows indicate the direction of the proposed high-velocity outflow from source I. The scale bar indicates 100 AU. An arrow as long as the bar corresponds to a motion of about $50 \mathrm{~km} \mathrm{~s}^{-1}$. The 1983 VLA observations covered a total velocity range of -12.4 to $28.1 \mathrm{~km} \mathrm{~s}^{-1}$, except for velocities close to a $4 \times 10^{6} \mathrm{Jy} \mathrm{H}_{2} \mathrm{O}$ maser flare 32 with a velocity of $8 \mathrm{~km} \mathrm{~s}^{-1}$ and a position of $\alpha_{2000}=5^{h} 35^{m} 14^{s} .121 \pm 0.003$, $\delta_{2000}=-05^{\circ} 22^{\prime} 36^{\prime \prime} .27 \pm 0.05$. The maser data were self-calibrated and relative positions for the maser spots measured. Absolute positions were obtained from low dynamic range images obtained contemporaneously by a wide-band antenna subarray that observed the maser flare and a phase calibrator, 0529+075.

1c A proposed model for the region surrounding source $\mathbf{I}$, showing the high-velocity and low-velocity outflows schematically as cones and a non-axisymmetric torus, respectively. The front surface of the torus is cut-away for clarity, and the $\mathrm{SiO}$ and $\mathrm{H}_{2} \mathrm{O}$ maser distributions 
are superposed. The arrows indicate schematically the high-velocity outflow; the arrows at position angles of $-60^{\circ}$ and $120^{\circ}$ also correspond approximately to the direction of the magnetic field in OMC-1 2 . A toroidal geometry is suggestive but not unique. The $\mathrm{H}_{2} \mathrm{O}$ masers lie close to the surface of the torus, probably in outward-propagating shocks. Overall, the zone in which the $\mathrm{H}_{2} \mathrm{O}$ masers lie may not move significantly over time, as individual shocks move outward and diminish over time, while new ones are born closer to the protostar. Otherwise, the dynamical age of the equatorial outflow is on the order of 100 years.

Figure 2 A near-infrared image $(3.8 \mu \mathrm{m})$ of the region near IRc2 27 , with contours representing $8.4 \mathrm{GHz}$ emission $\mathrm{b}^{\mathrm{a}}$ and the $\mathrm{H}_{2} \mathrm{O}$ maser distribution superposed. The bar indicates 1,000 AU. Labels mark the four components of IRc2 (A, B, C, and D) and other infrared sources. Specifically, source $\mathbf{n}$ is the brightest infrared object in the field. It is probably a young massive star with an associated HII regiona, though no dust emission have been detected yet at $3 \mathrm{~mm}$ wavelength 15 . The dashed box indicates the field of view in Figure 1b. The $\mathbf{X}$ highlights a possible relationship bewteen the proposed outflow from source $\mathbf{I}$ and IRc2. Also shown is the center of expansion (C.O.E.) for the low-velocity $\left(18 \mathrm{~km} \mathrm{~s}^{-1}\right)$ flow in $\mathrm{BN} / \mathrm{KL}$, estimated in part from the proper motions of $\mathrm{H}_{2} \mathrm{O}$ masers outside the field shown 12 . 


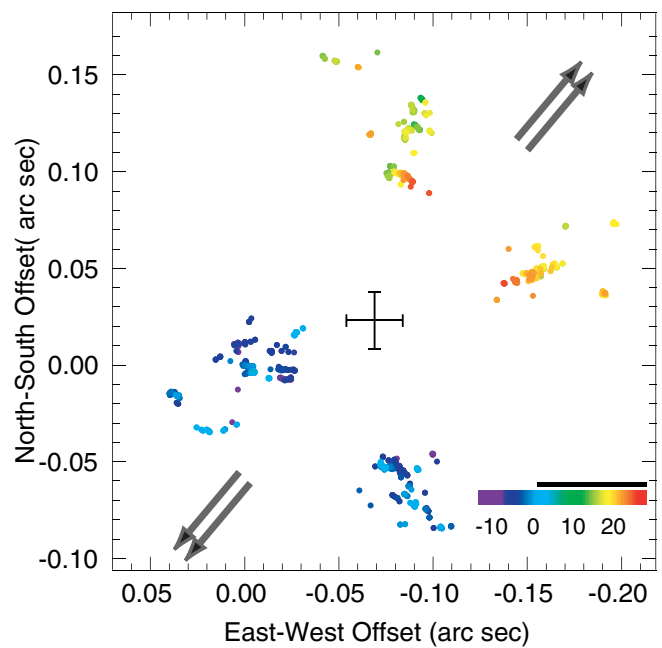

b

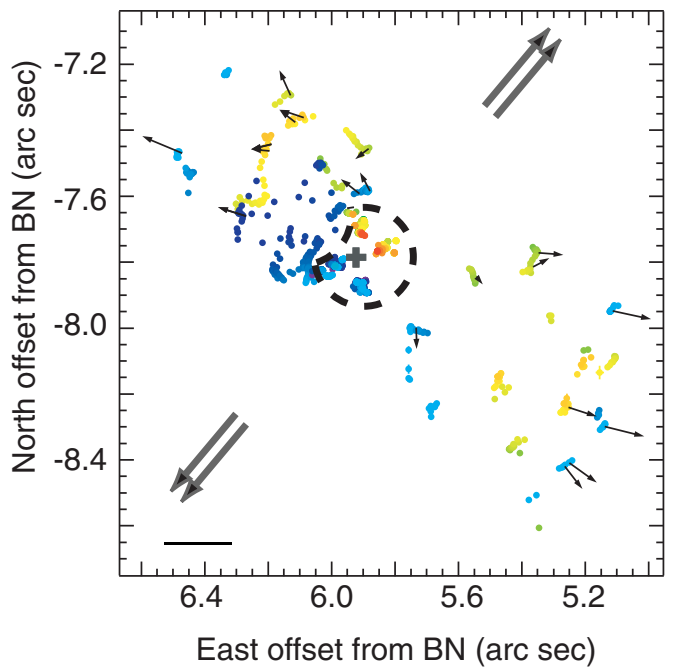

c

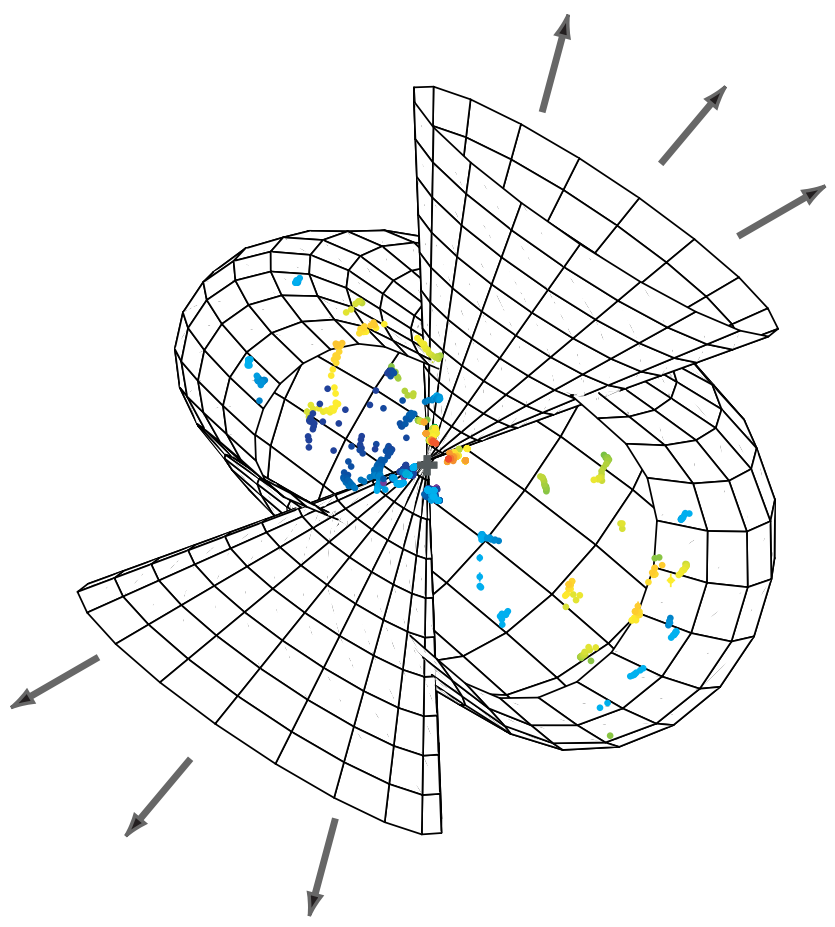

Figure 1

Greenhill et al. 


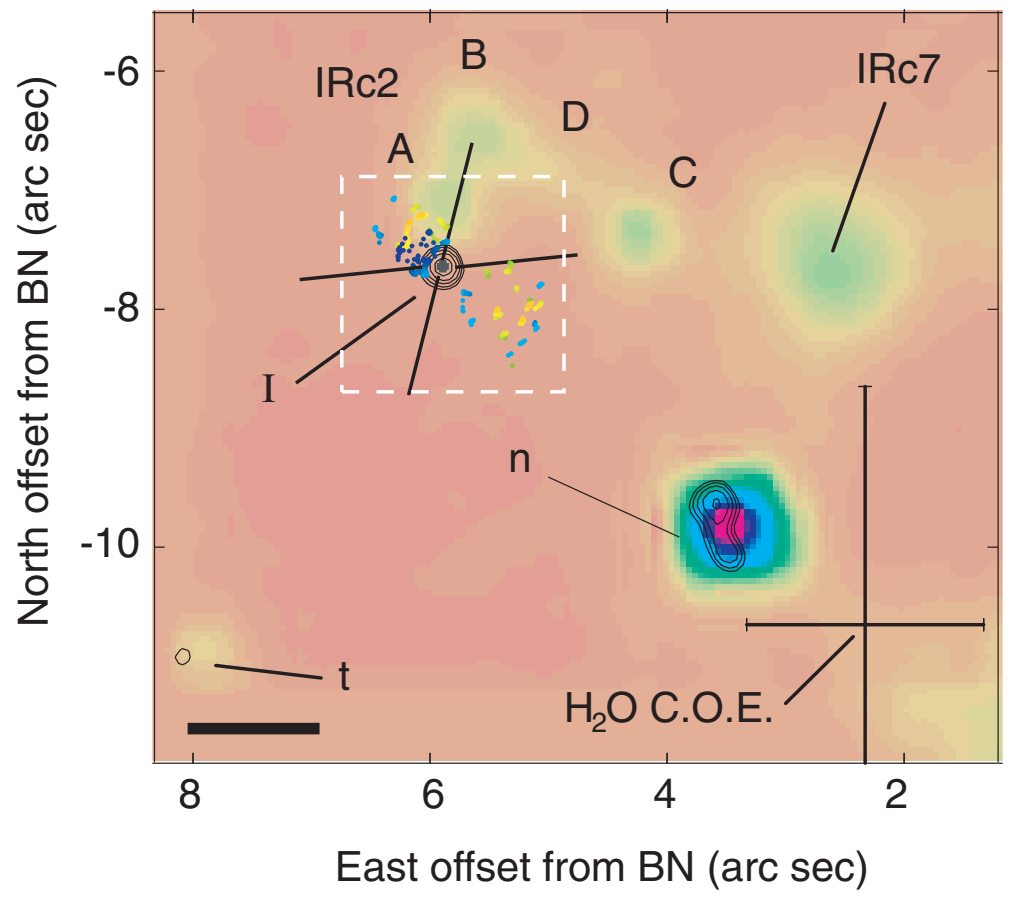

Greenhill et al. Figure 2 